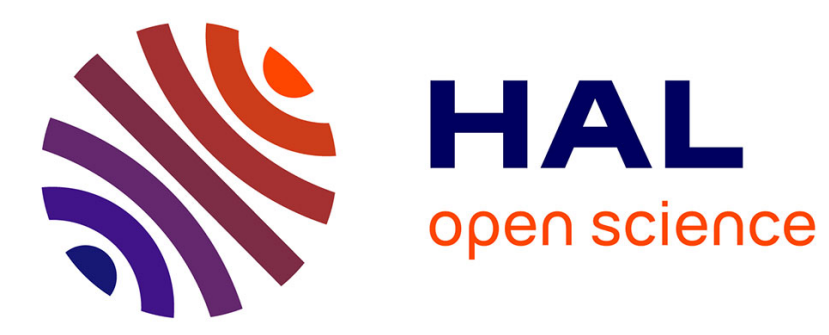

\title{
Experimental and numerical study of weak shock wave transmissions through minitubes
}

\author{
Jean-Denis Parisse, Laurent Biamino
}

\section{To cite this version:}

Jean-Denis Parisse, Laurent Biamino. Experimental and numerical study of weak shock wave transmissions through minitubes. Physics of Fluids, 2010, 10.1063/1.3432501 . hal-01446869

\section{HAL Id: hal-01446869 \\ https://hal.science/hal-01446869}

Submitted on 26 Jan 2017

HAL is a multi-disciplinary open access archive for the deposit and dissemination of scientific research documents, whether they are published or not. The documents may come from teaching and research institutions in France or abroad, or from public or private research centers.
L'archive ouverte pluridisciplinaire HAL, est destinée au dépôt et à la diffusion de documents scientifiques de niveau recherche, publiés ou non, émanant des établissements d'enseignement et de recherche français ou étrangers, des laboratoires publics ou privés. 


\title{
Experimental and numerical study of weak shock waves transmissions through mini-tubes
}

\author{
J. Giordano,* J. D. Parisse, L. Biamino, J. Devesvre, and P. Perrier \\ IUSTI UMR 6595 CNRS/Aix Marseille Université \\ Technopôle de Château Gombert, 5 rue Enrico Fermi, 13013 Marseille, France
}

(Dated: April 15, 2010)

\begin{abstract}
The aim of this paper is to present an original experimental technique to study weak shock wave in a mini-tube. Thus, we have designed an apparatus, which can be connected to any classical shock tube, in order to characterise high speed flows induced by the shock wave transmission in minitubes. We have proposed appropriated measurements based on high speed strioscopy coupled with pressure sensors. Two mini-tube diameters are considered: $1.020^{ \pm 0.010} \mathrm{~mm}$ and $0.480^{ \pm 0.010} \mathrm{~mm}$. We have realized preliminary experimental and numerical campaigns with an incident shock wave Mach number at $1.12^{ \pm 0.01}$. The generation of a micro-shock wave was observable in the two minitubes. For the smallest mini-tube, we have found an attenuation of the strength of the shock wave with a decrease of $1.8 \%$ of the Mach number.

PACS numbers: 47.61.-k, 47.40.-x, 47.11.Df
\end{abstract}

*Electronic address: jerome.giordano@polytech.univ-mrs.fr 
The recent researches in the fluids mechanics of micro-systems let us foresee the advances that may allow the miniaturization of micro-valves, micro-nozzles or micro-pumps [1]. In this micro-devices the ratio between lateral surfaces and volume could be extremely greater than in macro devices and, as consequence, increases the energy exchanges. If we focus on the domain of the micro-flows of gas, many studies have been proposed by the scientific community since the last two decades $[2-4]$. Previous studies were carried in our team to treat microfluidic flows with low velocity regime. The aim of these studies was to characterize the gas/wall interactions through the slip velocity (in the slip regime) and the estimation of accommodation coefficients from hydrodynamics to free molecular regime (Knudsen Number $[0.001,50])[5,6]$. Moreover, these studies have allowed us to develop experimental techniques to deal with micro-flows at low speed [7]. However, the applications in high speed regime are numerous, for example in micro-wave rotors where shock waves are efficiently used to transfer energy [8]. Nevertheless, the shock propagation in micro channel is not yet well studied and need to be better understood to increase micro-sytems efficiency. Even if we can note recent analytical modelization study, such as Mirshekari and Brouillette's one [9], there is an important lack of experiments dealing with the high speed flows, with or without shock wave. To our knowledge, the only working device devoted to the high speed gaseous flows in micro-channels is that developed by Udagawa, Garen and their co-workers in $[10,11]$. Their apparatus is based on the use of a quickly deformable opening valve which initially separates the micro channel from the high pressure chamber. In previous studies, we have numerically investigated the possibility to transmit a shock wave into a micro-channel [12]. In this work, several simulations have been realized to consider the influence of the microchannel dimensions. Besides, we have designed an original apparatus based on the use of a generic micro-valve to generate micro-shock waves $[13]$. However, before building this whole device, we have decided to use our classical shock tubes facilities in order to study weaker micro-shock waves and to bring additional validations for our code CARBUR [14] to deal with microfluidics phenomena. Thus, the present paper is dedicated to the presentation of the new experimental technique and the results we have obtained both experimentally and numerically. First of all, we describe how we have adapted a mini-tube to our classical shock tube T80 [15] and what types of measures have been done. Then, we present the results of experiments and simulations and we conclude and give the main perspectives of this work.

As the simplest device to generate shock wave is a shock tube, our idea is to connect a 
mini-tube to the end of a classical shock tube. A shock tube allows to study compressible flows with a good repeatability and with an easy control of the shock strength. With this principle, one can deal with the shock wave transmission and high speed flow generation in a micro-system. In this context, we use our T80 facility with a high speed shadowgraph video recording diagnostic. We use mirrors with a diameter equals to $0.3 \mathrm{~m}$ and a focale length of $3 \mathrm{~m}$. The video is recorded with the digital camera fastcam SA1 of Photron (San Diego - USA). All the experiments were made with Air, and because the mini-tube makes a connection between the shock tube and the ambient Air, the pressure $P_{1}$ was atmospheric pressure $1^{ \pm 0.01} \cdot 10^{5} \mathrm{~Pa}$ and the temperature was the ambient temperature $300^{ \pm 2} \mathrm{~K}$. In these conditions, the maximum of incident shock strength we can reach with our facility is equal to $M_{s}=1.5^{ \pm 0.01}$. One can note that is smaller than shock wave strength expected by the other device we have designed in [13]. Mini-tubes overall dimensions are given by the figure 1 . The mini-tubes are realized with commercial micro-syringes. We only keep the cylindrical glass tube of the micro-syringes, cut at the required dimensions. The main dimensions of mini-tubes are: for mini-tube 1, its length is equal to $81.24^{ \pm 0.05} \mathrm{~mm}$ and its diameter was measured at $1.020^{ \pm 0.010} \mathrm{~mm}$. For the mini-tube 2, its length is equal to $46.44^{ \pm 0.05} \mathrm{~mm}$ and its diameter measured at $0.480^{ \pm 0.010} \mathrm{~mm}$. As the figure 1 shows, the system was designed for an easy adaption of the different mini-tubes. The support is made in plexiglass and fixed to the shock tube ending. The fluid flow characterization is carried through two types of measurement. First of all, the macro-tube T80 is instrumented with several pressure sensors, regularly positioned along the tube. These sensors allow us to know the celerity of the incident shock wave. To obtain an estimation of the shock wave celerity in the mini-tube, another sensor is placed out of the device, right in front of the mini-tube (Fig. 1). Indeed, we cannot use classical pressure sensors along the wall of the mini-tube, as it is done in the macro-tube, without major flow disturbances. Nevertheless, the information given by this pressure sensor takes into account the sum phenomena which occurred in the mini-tube. Thus, this measure is relevant to give a validation for our simulations. A second measure is provided with a high speed shadowgraph digital video recording. The recording rate is fixed at $90000 \mathrm{fps}$ and we visualize the space near the mini-tube ending. These pictures are useful to identify the shock structures generated forward the mini-tube outlet and to determine the velocity of these shock wave structures. One can note that we have also realized experiments without the pressure sensor positioned in front of the mini-tube in order to study the free 
jet structures. We use our numerical code CARBUR [13] to simulate the compressible air fluid flows. This code is based on a second order discretization, in time and in space, of the Navier-Stokes' equations with a finite volume method. Convective fluxes are estimated through the solution of a Riemann's problem at each cell interface with an exact solver, whereas diffusive ones are obtained with a finite differences method. The walls are supposed as adiabatic with no velocity slip. The geometry is decomposed into 100000 cells densely distributed close to walls : $1.10^{-5} \mathrm{~m}$. In the two mini-tubes, 100 cells are used in the radial direction to describe correctly the velocity distribution.

For the two mini-tubes, we have realized a first campaign of simulations and experiments with an incident Shock wave Mach number at $\approx 1.12^{ \pm 0.01}$. In these first simulations, the flow was supposed as laminar. With these flow conditions, the Knudsen's number is lower than 7. $10^{-5}$ in the mini-tube 1 and lower than $1.410^{-4}$ in the mini-tube 2. As a consequence, the flow is in the continuous regime and the Navier-Stokes approach is fully relevant. These values of the Knudsen's number are small because we have chosen to work with high levels of pressure. So, we do not have to take into account rarefaction effects as it was done by Brouillette [3], Zeitoun and Burtschell [16]. The Reynold's number, calculated with the mean velocity, is close to 4400 along the mini-tube 1 and close to 2500 along the mini-tube 2. To quantify the influence of the turbulence on the transmitted shock wave into mini-tube 1, others simulations have been made with the Spalart-Allmaras turbulence model [17]. We found that the velocity of the transmitted shock wave is about $407 \mathrm{~m} . \mathrm{s}^{-1}$ in laminar assumption and about $408 \mathrm{~m} . \mathrm{s}^{-1}$ in turbulent one. This $1 \mathrm{~m} . \mathrm{s}^{-1}$ of deviation can be neglected compared to $10 \mathrm{~m} . \mathrm{s}^{-1}$ of the speed increase of the shock wave observed in this mini-tube 1. These results confirm that, in these experiment conditions, the turbulent effects have no significant influence on the transmitted shock wave velocity. Therefore, we think that turbulence effects can be neglected in our study of transmitted shock wave modifications by the micro-scales effects, even if the Reynolds number is close to 5000.

In the figure 2, one can see the time sequence of typical experimental Schlieren, pictures at every $22.22^{ \pm 0.1} \mu s$, observed when the transmitted shock wave and the flow get out the mini-tube. These pictures give an overview of the fluid flow generated downstream the minitube and allow us to identify a discontinuity followed by a jet stream with vortex structures. The figure 3 represents the main discontinuities and interactions of the fluid flow in a space time diagram (mini-tube 1 and incident shock wave at Mach $1.12^{ \pm 0.01}$ ). The lines are the iso- 
contour of the fluid flow temperature given by the simulations, whereas experimental points are provided by the different pressure sensors and the high speed camera. Each pressure sensors give one measure of pressure at one location per experiment, nevertheless we have plotted on the figure 3 the pressure measures from four experimental runs. For these different runs, variations of initial conditions are observed (ambient temperature and pressure, etc.) which induce a low variation of the fluid flow properties, particularly the Mach number of the incident shock wave. On the figure 3 , the results of one simulation are plotted. The fluid flow conditions of this simulation correspond to one experimental run whose measures are represented by the points (triangle for the pressure measure and square for the optic ones) filled with white. The other pressure measurements from the three other runs are filled with grey. One can note that experiments and simulation are in good agreement and, as a consequence, that simulations seem to describe correctly the main physics phenomena. The repeatability is correct as the pressure measures show, but should be improved in the future. Moreover, the experimental and numerical results attest that a shock wave is transmitted into the two mini-tubes. This transmitted shock wave is transmitted again into the air at rest at the mini-tube ending. The secondly transmitted wave is a blast wave with a celerity equals to the sound one. Following the acoustic wave, a stream flow is observable. The mean velocity of this jet is close to $110 \mathrm{~m} . \mathrm{s}^{-1}$ for the mini-tube 1 (less than $5 \mathrm{~m} . \mathrm{s}^{-1}$ of deviation between experiments and simulations). For the second mini-tube, this velocity is close to 105 m.s $\mathrm{s}^{-1}$ with less than 5 m.s $\mathrm{s}^{-1}$ of deviation between numerical and experimental results.

One can also make an important remark: the pressure sensor does not detect the acoustic wave but the jet stream. This is explained by the low pressure gradient observable across the acoustic wave which is estimated by simulation at less than $500 \mathrm{~Pa}$. The pressure sensor cannot detect this pressure jump due to its own detection limit around $5000 \mathrm{~Pa}$. Besides, we have estimated the Mach number of transmitted shock wave for each mini-tube. For the mini-tube 1 (diameter equal to $1.020^{ \pm 0.010} \mathrm{~mm}$ ), the transmitted shock wave Mach number is close to $1.15^{ \pm 0.01}$ (mean estimated along $80 \times$ diameters), which represents an increasing of $\approx 2.5 \%$. For the second mini-tube (diameter equal to $0.480^{ \pm 0.010} \mathrm{~mm}$ ), we note a decrease of the transmitted shock wave Mach number of $\approx 1.8 \%$ (Mach equals to $\approx 1.10^{ \pm 0.01}$, mean estimated along $80 \times$ diameters). So, these results seem to confirm the micro-scale influences on the shock wave strength previously observed by Parisse et al. in [12]: the decrease of the mini-tube diameter induces a the diminution of the shock wave celerity. What is remarkable 
is that, in spite of the section changing between the macro-tube and mini-ones which should lead to the increasing of the shock wave strength [18], we observe in the mini-tube 2 a weaker shock wave. This shock wave attenuation effect is explained by the growth of the boundary layer, which at these scales quickly modifies the idealized wave system and the flow variables. For the mini-tube 1, an attenuation should be visible for a greater length than $80 \times$ diameters.

To check the viability of our device to study higher incident shock wave Mach numbers, we have realized one run at Mach $\approx 1.5^{ \pm 0.01}$. The pictures $(\mathrm{a})$ and $(\mathrm{b})$ of the figure 4 show the comparison of a numerical and experimental schlieren for the flow generated upstream the mini-tube 1 . On this figure, we can identify the acoustic radiations induced by the vortex structure of the jet. The frequency of acoustic wave emission is about $165 \mathrm{KHz}$. An estimation of the mechanical eigen-frequencies of the apparatus (software CATIA, Dassault System, Vélizy-Villacoublay - France) shows that the mechanical vibrations are not coupled with the vortex shedding and acoustic wave emission. Even if simulation and experiment are in relatively good agreement for the early times, for this stronger shock wave, the turbulence has to take into account for describe the jet-stream development. Indeed, as we can see in the picture (c) of the figure 4, the jet-stream becomes shortly fully turbulent. We can see that our experimental apparatus could be used to characterize the behaviour of high speed micro-jets. The knowledge of these micro-jets should be useful for the safety of high pressurized tanks. Indeed, a micro leak could have the same structures as the micro-jet observed with our experiments.

In conclusion, we have designed and built an original device to study micro-compressiblefluid flows. This experimental apparatus could be easily adapted in a classical shock tube, as we have done. We have realized first campaigns of experiments to try to transmit an incident Shock Wave at Mach $1.12^{ \pm 0.01}$ into two mini-tubes. In a same time, we have simulated these experiments with our code CARBUR and, after different comparisons, we have found a good agreement between the two approaches. Thus, we have seen, in all the cases treated in this study, that a shock wave was transmitted into the mini-channel. For the smallest minitube, we have found an attenuation of the strength of the shock wave with a decrease of $1.8 \%$ of the Mach number. To our knowledge, the measure of this shock attenuation for these scales (475 $\mu \mathrm{m}$ for mini-tube 2$)$ and for these pressure level $\left(P_{1}=1^{ \pm 0.01} .10^{5} \mathrm{~Pa}\right.$ ) has not been provided experimentally before. Thus, these first results are promising, and more 
experiments for different shock wave strengths should be realized to confirm these primary conclusions. Moreover, different perspectives could be considered. On the one hand, we could decrease the initial pressure in the mini-tube to study rarefaction effects. On the other hand, we could also use different gases, like Helium/Air or Helium/Argon, to reach higher shock wave Mach number and as a consequence higher compression effects. Besides, combustion phenomena could be also investigated for example by using mixture of $\mathrm{H}_{2}$ and $\mathrm{O}_{2}$ gases.

[1] D. J. Laser and J. G. Santiago, "A review of micropumps ", Journal of Micromechanics and Microengineering, 14, R35, (2004).

[2] M. Sun, T. Ogawa and K. Takayama, "Shock propagation in narrow channel", Proc. of 23rd Int. Symposium on Shock waves Fort Worth, Texas USA, (2002).

[3] M. Brouillette, "Shock waves at microscales", Shock Waves Journal, 13, 3, (2004).

[4] L. O'Hare, D. A. Lockerby, J. M. Reese and D. R. Emeron, "Near-wall effects in rarefied gas micro flows: some modern hydrodynamics approaches", International Journal of Heat and Fluid Flow, 28, 37, (2001).

[5] T. Ewart, P. Perrier, I. Graur and J. G. Molans, "Mass flow rate measurements in microchannel, from hydrodynamic to near free molecular regimes", Journal of fluid mechanics, 584, 337 (2007).

[6] T. Ewart, P. Perrier, I. Graur and J. G. Molans, "Tangential momentum accomodation in microtube", Microfluidics and Nanofluidics, 3, 689, (2007).

[7] T. Ewart, P. Perrier, I. Graur and J. G. Molans, "Mass flow rate measurements in gas micro flows", Experiments in Fluids, 41, 487, (2006).

[8] F. Iancu, X. Zhu, Y. Tang, D. Alsam and N. Mller, "Design and fabrication of microchannel test rig for ultre-micro wave rotors", Microsystem Technologies, 14, 79, (2008).

[9] G. Mirshekari and M. Brouillette, "One-dimensional model for microscale shock tube flow", Shock Waves Journal, 19, 25, (2009).

[10] S. Udagawa and W. Garen and B. Meyerer and K. Maeno, "Interferometric detection of dispersed shock waves in small scale diaphragm-less shock tube of $1 \mathrm{~mm}$ diameter", 16th Australasian Fluid Mechanics Conference, Brisbane Australia, School of Engineering, The 